\title{
Analysis of De Novo Mutations in Sporadic Cardiomyopathies Emphasizes Their Clinical Relevance and Points to Novel Candidate Genes
}

\author{
Maria Franaszczyk ${ }^{1}{ }^{1}$, Grazyna Truszkowska ${ }^{1}$, Przemyslaw Chmielewski ${ }^{2}$, \\ Malgorzata Rydzanicz ${ }^{3}{ }^{\circledR}$, Joanna Kosinska ${ }^{3}$, Tomasz Rywik ${ }^{4}$, Anna Biernacka ${ }^{5}$, \\ Mateusz Spiewak ${ }^{6}{ }^{\oplus}$, Grazyna Kostrzewa ${ }^{7}$, Malgorzata Stepien-Wojno ${ }^{2}$, \\ Piotr Stawinski ${ }^{3}$, Maria Bilinska ${ }^{8}$, Pawel Krajewski ${ }^{7}$, Tomasz Zielinski ${ }^{4}{ }^{(}$, \\ Anna Lutynska ${ }^{9}$, Zofia T. Bilinska ${ }^{2, *(1)}$ and Rafal Ploski ${ }^{3, *(1)}$
}

1 Molecular Biology Laboratory, Department of Medical Biology, Institute of Cardiology, 04-628 Warsaw, Poland; m.fran@wp.pl (M.F.); gtruszkowska@ikard.pl (G.T.)

2 Unit for Screening Studies in Inherited Cardiovascular Diseases, Institute of Cardiology, 04-628 Warsaw, Poland; pchmielewski@ikard.pl (P.C.); mstepien@ikard.pl (M.S.-W.)

3 Department of Medical Genetics, Medical University of Warsaw, 02-106 Warsaw, Poland; mrydzanicz@wum.edu.pl (M.R.); samocko@wp.pl (J.K.); stawinski84@gmail.com (P.S.)

4 Department of Heart Failure and Transplantology, Institute of Cardiology, 04-628 Warsaw, Poland; rywikt@ikard.pl (T.R.); tzielinski@ikard.pl (T.Z.)

5 Postgraduate School of Molecular Medicine, Medical University of Warsaw, 02-091 Warsaw, Poland; biernackann@gmail.com

6 Magnetic Resonance Unit, Department of Radiology, Institute of Cardiology, 04-628 Warsaw, Poland; mspiewak@ikard.pl

7 Department of Forensic Medicine, Medical University of Warsaw, 02-007 Warsaw, Poland; g.kostrzewa@wp.pl (G.K.); pawel.krajewski@wum.edu.pl (P.K.)

8 Department of Arrhythmia, Institute of Cardiology, 04-628 Warsaw, Poland; mbilinska@ikard.pl

9 Department of Medical Biology, Institute of Cardiology, 04-628 Warsaw, Poland; alutynska@ikard.pl

* Correspondence: zbilinska@ikard.pl (Z.T.B.); rploski@wp.pl (R.P.); Tel.: +48-223434710 (Z.T.B.); +48-225720695 (R.P.)

Received: 10 December 2019; Accepted: 24 January 2020; Published: 29 January 2020

\begin{abstract}
The vast majority of cardiomyopathies have an autosomal dominant inheritance; hence, genetic testing is typically offered to patients with a positive family history. A de novo mutation is a new germline mutation not inherited from either parent. The purpose of our study was to search for de novo mutations in patients with cardiomyopathy and no evidence of the disease in the family. Using next-generation sequencing, we analyzed cardiomyopathy genes in 12 probands. In $8(66.7 \%)$, we found de novo variants in known cardiomyopathy genes (TTN, DSP, SCN5A, TNNC1, TPM1, $C R Y A B, M Y H 7)$. In the remaining probands, the analysis was extended to whole exome sequencing in a trio (proband and parents). We found de novo variants in genes that, so far, were not associated with any disease (TRIB3, SLC2A6), a possible disease-causing biallelic genotype (APOBEC gene family), and a de novo mosaic variant without strong evidence of pathogenicity (UNC45A). The high prevalence of de novo mutations emphasizes that genetic screening is also indicated in cases of sporadic cardiomyopathy. Moreover, we have identified novel cardiomyopathy candidate genes that are likely to affect immunological function and/or reaction to stress that could be especially relevant in patients with disease onset associated with infection/infestation.
\end{abstract}

Keywords: cardiomyopathy; genetic; de novo mutation; novel genes 


\section{Introduction}

Cardiomyopathies are a group of diseases defined as structural and functional abnormalities in the cardiac muscle that cannot be explained by abnormal loading conditions [1]. Cardiomyopathies have a well-established genetic background and their genetic heterogeneity is characterized by the overlapping of disease genes for different clinical types [2]. The majority of cardiomyopathies have an autosomal dominant inheritance pattern. Therefore, genetic testing is typically offered to patients with a positive family history, whereas sporadic cases may mistakenly be classified as acquired without considering the potential role of de novo mutations. This is particularly important considering pediatric cases with no family history.

A de novo mutation is a new germline mutation caused by mutagenesis occurring in parental gametes, thus not inherited from either parent. Such a genetic variant occurs for the first time in one family member, thus forming the first generation of carriers, who can then transmit this new variant to their offspring.

It should be noted that the majority of cardiomyopathies causing de novo mutations have been reported in syndromic cases such as those caused by variants in DSP (Naxos-Carvajal syndrome [3], erythrokeratodermia-cardiomyopathy syndrome [4]), LAMP2 (Danon disease [5]), PRKAG2 (glycogen storage disease of heart [6,7]), RAF1 (Noonan syndrome [8]), TAZ (Bart syndrome [9]), RRAGC (syndromic fetal dilated cardiomyopathy [10]), and LMNA (atypical progeroid syndrome and dilated cardiomyopathy [11]). Data on de novo mutations in non-syndromic cardiomyopathies are limited and include variants in ACTC [12], MYH7 [13-16], TNNI3 [17], TNNT2 [18,19], and TPM1 [20,21].

The purpose of our study was to search for de novo mutations in patients with cardiomyopathy and no evidence of the disease in the family after a detailed clinical evaluation of proband-parent trios and, if available, other relatives.

\section{Materials and Methods}

Criteria for inclusion in the study required a pattern of the disease in the given family consistent with the possibility of a de novo mutation. Clinical examination of probands with cardiomyopathy included 12-lead electrocardiography (ECG), two-dimensional Doppler echocardiography, coronary angiography or coronary computed tomography angiography (CTA), serum creatine phosphokinase (CPK) and, if clinically indicated, cardiac magnetic resonance (CMR). The criteria for parents were clinically examined and healthy (based on normal non-invasive cardiac examination - ECG, echocardiogram), both living or at least with well-documented non-cardiac death and available genetic material.

Dilated cardiomyopathy was defined according to European Society of Cardiology (ESC) criteria: Left ventricular ejection fraction (LVEF) below $45 \%$ and left ventricular end-diastolic diameter (LVEDD) $>117 \%$ of the predicted value, corrected for age and body surface area [1].

Left ventricular noncompaction was diagnosed based on CMR study with the ratio of noncompacted to compacted myocardium greater than 2.3 during diastole on long-axis cine images [22].

Hypertrophic cardiomyopathy was defined as left ventricular hypertrophy in the absence of loading conditions, sufficient to account for the observed degree of hypertrophy, with a maximal left ventricular wall thickness $\geq 15 \mathrm{~mm}$ in one or more myocardial segments [1].

Diagnostic criteria for restrictive cardiomyopathy were restrictive ventricular physiology in the presence of normal or reduced diastolic volumes (of one or both ventricles), normal or reduced systolic volumes, and normal ventricular wall thickness [1].

None of the probands had hypertension, diabetes, or clinically significant atherosclerosis. For parents, these morbidities (when the criteria for cardiomyopathy were not met) were not considered an exclusion criterion.

The study was approved by the local Bioethics Committee of the Institute of Cardiology with approval numbers 1276 and 1451. All patients examined in the study signed written informed consent in accordance with the Declaration of Helsinki. Genetic testing and blood sampling were offered 
to all probands and relatives who agreed to participate in the study. All proband-parent trios have undergone both paternity and maternity testing.

DNA was extracted from the peripheral blood by phenol extraction or the salting-out method. In the probands, we performed next-generation sequencing including whole exome sequencing $(n=10)$, TruSight One panel $(n=1)$, and TruSight Cardio panel $(n=1)$, (Illumina, San Diego, CA, USA). Whole exome sequencing (WES) libraries were constructed using the TruSeq Exome Enrichment Kit (Illumina), Nextera Rapid Capture Exome Kit (Illumina), SeqCap EZ MedExome kit (Roche, Basel, Switzerland), and SureSelectXT Human All Exon v5 (Agilent Technologies, Santa Clara, CA, USA) according to the manufacturers' instructions and analyzed as described previously [23]. TruSight One and TruSight Cardio panel sequencing were performed according to the manufacturer's instructions. All libraries were pair-end sequenced on HiSeq 1500 or MiSeq (Illumina). A minimum of $20 \times$ coverage was obtained for the mean of $81 \% \pm 13$ of target regions. The results were visualized using Integrative Genomics Viewer v.2.3.81 (IGV, http://software.broadinstitute.org/software/igv/).

Parenthood was confirmed by the Forensic Medicine Department of the Medical University of Warsaw. DNA samples were short tandem repeats (STR) genotyped using PowerPlex Fusion 6C (Promega, Madison, WI, USA).

Baseline analysis of next generation sequencing (NGS) results consisted of searching for genetic variants with very-low-frequency $(<0.001)$ and high-bioinformatic-pathogenicity prediction scores with special regard to phenotypically plausible genes. The frequencies of variants were derived from gnomAD (http://gnomad.broadinstitute.org), NHLBI GO Exome Sequencing Project (ESP) 6500 (https: //esp.gs.washington.edu/drupal), and an in-house database of $>1000$ Polish subjects examined by WES. For the bioinformatic prediction scores, we used data summarized in VarSome (https://varsome.com). The clinical significance of the variants was based on ClinVar (https://www.ncbi.nlm.nih.gov/clinvar).

If a potentially damaging variant in one of the known genes associated with cardiomyopathies was found in the proband, instead of performing WES for a trio, a more cost-effective approach was used to confirm or exclude the presence of variants of interest identified by NGS. We performed Sanger sequencing in a trio setting (proband-parent) and, if available, in other relatives. For Sanger sequencing, we used the 3500xL Genetic Analyzer (Life Technologies, Carlsbad, CA, USA) and BigDye Terminator v3.1 Cycle Sequencing Kit (Life Technologies) following the manufacturer's instructions. Chromatograms were analyzed using Variant Reporter 1.1 (Life Technologies). Finding a de novo mutation in a cardiomyopathy-associated gene ended the diagnostic process.

In the remaining cases, with no candidate variant found using the strategy described above, WES was also carried out for the proband's parents. Further analysis was performed as described above (including bioinformatic analysis and variant Sanger sequencing) with focus on rare variants present in the proband but absent in the parents. Additionally, copy number variants $(\mathrm{CNV})$ analysis was performed using the CNVkit [24] with a reference population of around 30 samples.

\section{Results}

We identified 12 probands with sporadic cardiomyopathy who fulfilled the abovementioned criteria. The pedigrees are shown in corresponding Figure 1, Figure 2, and Figure S1, and clinical characteristics are given in Table S2. Ten patients had dilated cardiomyopathy (DCM), reflecting a relatively high frequency of sporadic cases in this condition (families FD01-10). Two DCM patients additionally had left ventricular noncompaction (LVNC). One patient had an atypical course of hypertrophic cardiomyopathy (HCM) with restrictive features (family FH1) and suspicion of storage disease, and one fulfilled the criteria for restrictive cardiomyopathy (RCM) (family FR1).

\subsection{De Novo Mutations in Known Cardiomyopathy Genes}

In $8 / 12$ probands $(66.7 \%)$, we found likely causative variants in known cardiomyopathy genes (TTN, DSP, SCN5A, TNNC1, TPM1, CRYAB, and MYH7), which were confirmed as de novo (six DCM, one HCM, and one RCM case, Table 1, Figure S1). Considering only DCM, the prevalence of de novo 
mutations in the known genes was 60\% (6/10). Genetic findings and genotype-phenotype correlations are described in detail below (see also Table 1 and Table S2).

\subsubsection{Family FD02}

In the proband, we found a de novo missense mutation p.Trp976Leu/c.2927G $>\mathrm{T}$ in the TTN gene (titin, MIM \#188840). The mutation was absent in the proband's parents and two sisters but inherited by her affected son. The variant has high deleteriousness scores (seven pathogenic predictions vs. no benign predictions) and was not found in gnomAD. Moreover, mutation in the same amino acid (p.Trp976Arg, rs267607155) was described by Gerull et al. [25] as segregating with DCM in a three-generation large kindred, and its protein-damaging effect was confirmed in a functional study [26].

The 34-year-old female proband was referred following diagnosis of DCM in her son. She was in New York Heart Association (NYHA) class II, in sinus rhythm, and, on the echocardiogram, had a dilated spherical left ventricle (LVEDD of $70 \mathrm{~mm}$ ), LVEF of $25 \%$, and restrictive mitral inflow pattern. No significant arrhythmia was found on the 24 hour Holter ECG. She had a history of recurrent tonsillitis. At the age of 30, she gave vaginal birth to a male child, in whom DCM was diagnosed at the age of three, following chickenpox. No other relatives had any cardiac abnormalities.

\subsubsection{Family FD03}

In the proband, we found a de novo missense mutation p.Glu290Lys/c.868G $>\mathrm{A}$ in the DSP gene (desmoplakin, MIM \#125647). The variant has high deleteriousness scores (nine pathogenic predictions vs. no benign predictions) and is absent from gnomAD. The same variant was described in a patient with arrhythmogenic right ventricular cardiomyopathy (ARVC), repolarization abnormalities, and family history, although no functional or segregation studies were performed [27].

The female proband started experiencing palpitations at the age of $26 \mathrm{y}$, and was diagnosed with DCM at $28 \mathrm{y}$. CMR showed impaired left ventricle (LV) function (LVEF 37\%), late gadolinium enhancement (LGE), and normal right ventricle. Repeated Holter ECGs revealed frequent and complex ventricular arrhythmia including bursts of non-sustained ventricular tachycardia (nsVT), and a cardioverter-defibrillator (ICD) was implanted at the age of $30 \mathrm{y}$. The patient has a history of thyrotoxicosis due to autoimmune thyroid nodular disease that could aggravate her symptoms and was vigorously treated with thyrostatics. However, the complex ventricular arrhythmia and impaired left ventricle function also persisted during euthyreosis.

\subsubsection{Family FD04}

In the proband, we found a de novo missense mutation p.Glu1548G/n/c.4642G $>C$ in the SCN5A gene (sodium voltage-gated channel alpha subunit 5, MIM \#600163). The variant has high deleteriousness scores (nine pathogenic predictions vs. no benign predictions) and was not found in gnomAD. Another variant in the same amino acid (p.Glu1548Lys/c.4642G>A) was described in three unrelated patients with Brugada syndrome [28].

The male proband was diagnosed because of palpitations at the age of $15 \mathrm{y}$. Since then, a variety of ventricular and supraventricular arrhythmias were identified, which led to radiofrequency ablation (RFA) of ventricular arrhythmia at the age of 17 and $22 \mathrm{y}$, and cavotricuspid isthmus-dependent atrial flutter ablation at the age of $22 \mathrm{y}$. At $23 \mathrm{y}$, his left ventricular function is mildly depressed.

\subsubsection{Family FD06}

In the proband, we found a de novo missense mutation p.Glu94Val/c.281 $\mathrm{A}>\mathrm{T}$ in the TNNC1 gene (troponin C1, slow skeletal and cardiac type, MIM\#191040). The variant has high deleteriousness scores (eight pathogenic predictions vs. one benign prediction) and was not found in gnomAD. Another variant in the same amino acid (p.Glu94Ala/c.281A $>$ C) was described in a patient with LVNC with onset at $4 \mathrm{mo}$ and no family history who underwent heart transplant [29]. 
The 20-year-old male proband started having decreased exercise tolerance at 13 y. At 16 $\mathrm{y}$, the patient suffered cardiogenic shock, had a dilated left ventricle of $75 \mathrm{~mm}$ and LVEF of $15 \%$, and received a heart transplant after a bridge with a biventricular assist device (BiVAD) for three months.

\subsubsection{Family FD09}

In the proband, two missense candidate mutations were found: p.Ile201Thr/c.602T $>\mathrm{C}$ in the MYH7 gene (myosin heavy chain 7, MIM \#160760) and p.Lys205Arg/c.614A>G in the TPM1 gene (tropomyosin 1, MIM \#191010). The MYH7 variant has ambiguous deleteriousness scores (two pathogenic predictions vs. four benign predictions) while the TPM1 variant has high deleteriousness scores (six pathogenic predictions vs. one benign prediction). Neither variant was found in gnomAD. The family study revealed that the $M Y H 7$ variant arose de novo, while the TPM1 variant was inherited from the proband's asymptomatic father. The $M Y H 7$ variant has also been described as "likely pathogenic" in a DCM patient [30].

The 19-year-old male proband was diagnosed with DCM and LVNC after echocardiographic and CMR study performed due to palpitations. LVEF was 26\%, LVEDD was $62 \mathrm{~mm}$, and the ratio of noncompacted to compacted myocardial layer was 3.2:1. The patient received standard heart failure treatment, and at $24 \mathrm{y}$, his left ventricle function remained reduced (LVEF 37\%).

\subsubsection{Family FD10}

In the proband, we found a de novo missense mutation p.Thr40Met/c.119C $>\mathrm{T}$ in the $C R Y A B$ gene (alpha B-crystallin, MIM \#123590). The variant was inherited by the proband's daughter. The variant has ambiguous deleteriousness scores (three pathogenic predictions vs. four benign predictions) and was found in gnomAD $(\mathrm{f}=0.00001073)$.

The 44-year-old male proband suffered from an ischemic stroke at the age of $36 \mathrm{y}$ and, at that time, was diagnosed with DCM, LVNC, paroxysmal atrial fibrillation, intraventricular conduction disease (QRS of $153 \mathrm{~ms}$ ), and heart failure. Following standard heart failure treatment and with ICD implantation, he recovered substantially; however, eight months later, he had acute third-degree atrioventricular block and his ICD was upgraded to a cardiac resynchronization therapy defibrillator (CRT-D). The patient has a visual impairment with a short-sightedness of $-4.5 \mathrm{D}$ and astigmatism. His asymptomatic 20-year-old daughter has nonspecific ST-T changes in lateral leads on a 12-lead ECG and trabeculations in the inferolateral wall of the left ventricle, not meeting criteria for the diagnosis of noncompaction. She is also a short-sighted person.

\subsubsection{Family FR1}

In the proband, two missense candidate mutations were found: p.Gly768Arg/c.2302G >A in $M Y H 7$ and p.Pro1066Arg/c.3197C >G in MYBPC3. Both variants have high deleteriousness scores: $M Y H 7$ has eight pathogenic predictions vs. no benign predictions, while the $M Y B P C 3$ variant has seven pathogenic predictions vs. one benign prediction, and neither was found in gnomAD. MYH7 p.Gly768Arg was previously described in a family with HCM [31]. The family study showed that the MYH7 variant was de novo, while the MYBPC3 variant was inherited from the father who did not have DCM.

The male proband was diagnosed at 28 y because he wanted to apply for a boating license, and his standard ECG was abnormal. Subsequent imaging studies including an echocardiogram, CMR, and angio-CT scan revealed a hypoplastic left ventricle with reduced diastolic volume and narrow entire aorta, and a hypertrophied right ventricle, most probably due to pulmonary hypertension. Normal or reduced diastolic volumes of the left ventricle, that resemble hypoplastic left heart, fit well into the definition of restrictive cardiomyopathy [1]. On the other hand, the narrow aorta corresponds well to the diminished size of the left ventricle. As, clinically, his disease resembled restrictive cardiomyopathy with high pulmonary hypertension, we assumed that exclusion of the other congenital abnormalities in our patient and the presence of the sarcomeric gene mutation allowed us to consider the diagnosis of restrictive cardiomyopathy in the patient. 
Table 1. Molecular characteristics of variants of interest in known cardiomyopathy genes found in probands.

\begin{tabular}{|c|c|c|c|c|c|c|c|c|}
\hline Family & NGS Target & Gene/Transcript & $\begin{array}{c}\text { Variant } \\
\text { (dbSNP ID) }\end{array}$ & $\begin{array}{c}\text { Genomic Coordinates } \\
\text { (GRCh38) }\end{array}$ & $\begin{array}{c}\text { Allele } \\
\text { Frequency }\end{array}$ & $\begin{array}{c}\text { ACMG Verdict } \\
\text { (default/if de novo) }\end{array}$ & $\begin{array}{l}\text { ClinVar Clinical } \\
\text { Significance }\end{array}$ & Status \\
\hline FD02 & WES & $\begin{array}{c}\text { TTN/ } \\
\text { NM_001267550.2 }\end{array}$ & $\begin{array}{l}\text { p.Trp976Leu/c.2927G }>\mathrm{T}^{1} \\
\text { (ND) }\end{array}$ & 2:178782979-C>A & 0 & VUS/ Likely Pathog & ND & Novel \\
\hline FD03 & WES & DSP/ NM_004415.2 & $\begin{array}{c}\text { p.Glu290Lys/c.868G }>\mathrm{A}^{1} \\
(\mathrm{rs} 397516974)\end{array}$ & 6:7565449-G>A & 0 & VUS/ Likely Pathog & VUS (1x) & Described [27] \\
\hline FD04 & TSC & $\begin{array}{c}\text { SCN5A/ } \\
\text { NM_198056.2 }\end{array}$ & $\begin{array}{l}\text { p.Glu1548Gln/c.4642G>C }{ }^{1} \\
\text { (ND) }\end{array}$ & 3:38554450-C>G & 0 & VUS/ Likely Pathog & ND & Novel \\
\hline FD06 & WES & $\begin{array}{c}\text { TNNC1/ } \\
\text { NM_003280.2 }\end{array}$ & $\begin{array}{l}\text { p.Glu94Val/c.281A }>\mathrm{T}^{1} \\
\text { (ND) }\end{array}$ & 3:52451780-T>A & 0 & VUS/ Likely Pathog & ND & Novel \\
\hline \multirow{2}{*}{ FD09 } & \multirow{2}{*}{ WES } & $\begin{array}{c}\text { MYH7/ } \\
\text { NM_000257.3 } \\
\end{array}$ & $\begin{array}{c}\text { p.Ile201Thr/c.602T>C }{ }^{1} \\
\text { (rs397516258) }\end{array}$ & 14:23431798-A>G & 0 & $\begin{array}{l}\text { Likely Pathog/ } \\
\text { Pathog }\end{array}$ & Likely Pathog $(3 \times)$ & Described [30] \\
\hline & & $\begin{array}{c}\text { TPM1/ } \\
\text { NM_001018006.1 }\end{array}$ & $\begin{array}{l}\text { p.Lys205Arg/c.614A }>\mathrm{G}^{2} \\
\text { (ND) }\end{array}$ & $15: 63061248-A>G$ & 0 & VUS/ Likely Pathog & ND & Novel \\
\hline FD10 & TSO & $\begin{array}{c}\text { CRYAB/ } \\
\text { NM_001885.2 }\end{array}$ & $\begin{array}{l}\text { p.Thr40Met/c.119C }>\mathrm{T}^{1} \\
\text { (rs782122417) }\end{array}$ & 11:111911606-G>A & $\begin{array}{c}1.07 \mathrm{e}-05 \\
(\mathrm{GnomAD})\end{array}$ & VUS/ Likely Pathog & ND & Novel \\
\hline \multirow{2}{*}{ FR1 } & \multirow{2}{*}{ WES } & $\begin{array}{c}\text { MYH7/ } \\
\text { NM_000257.3 }\end{array}$ & $\begin{array}{c}\text { p.Gly768Arg/c.2302G }>\mathrm{A}^{1} \\
\text { (rs727503260) }\end{array}$ & $14: 23425403-\mathrm{C}>\mathrm{T}$ & 0 & Pathog/Pathog & Pathog $(1 \times)$ & Described [31] \\
\hline & & $\begin{array}{c}\text { MYBPC3/ } \\
\text { NM_000256.3 }\end{array}$ & $\begin{array}{l}\text { p.Pro1066Arg/c.3197C > } \mathrm{G}^{2} \\
\text { (ND) }\end{array}$ & 11:47333327-G>C & 0 & VUS/ Likely Pathog & ND & Novel \\
\hline FH1 & WES & $\begin{array}{c}\text { MYH7/ } \\
\text { NM_000257.3 }\end{array}$ & $\begin{array}{c}\text { p.Arg453Cys } / \mathrm{c} .1357 \mathrm{C}>\mathrm{T}^{1} \\
\text { (rs121913625) }\end{array}$ & 14:23429005-G>A & 0 & $\begin{array}{l}\text { Likely Pathog/ } \\
\text { Pathog }\end{array}$ & $\begin{array}{c}\text { Pathog }(10 \times) \\
\text { Likely Pathog }(1 \times)\end{array}$ & $\begin{array}{l}\text { Described } \\
\text { [32-34] }\end{array}$ \\
\hline
\end{tabular}

${ }^{1}$ Variants verified as de novo. ${ }^{2}$ Variants also considered as disease-causing de novo candidate mutations but verified during a subsequent family segregation study as of parental origin. Allele frequency - allele frequency in gnomAD, 1000G P3, and ESP6500. WES - whole exome sequencing, TSC - TruSight Cardio; TSO - TruSight One, VUS - variant of uncertain significance, Pathog - pathogenic, ND - no data. 


\subsubsection{Family FH1}

In the proband, a de novo missense mutation p.Arg453Cys/c.1357C > T in the MYH7 gene was identified. The variant has high deleteriousness scores (eight pathogenic predictions vs. no benign predictions) and was not found in gnomAD. This $M Y H 7$ variant has established pathogenicity - it was described in patients with HCM [32,33] and DCM [34] and associated with poor prognosis.

The 39-year-old female proband started having exertional dyspnoea at $16 \mathrm{y}$ and was diagnosed with HCM. At $32 \mathrm{y}$, she had ankle oedema following second delivery. Her heart failure symptoms progressed, and at $34 \mathrm{y}$ on an echocardiogram, she was found to have asymmetric nonobstructive hypertrophic cardiomyopathy with reduced left ventricle function and prominent restrictive features. She received a heart transplant at $35 \mathrm{y}$.

\subsection{Novel DCM Candidate Genes - Findings from WES Analysis in Trios}

WES analysis was performed in four proband-parent trios (all probands with DCM) without mutation in any genes related to cardiomyopathy. In two probands, it revealed de novo variants in genes that were, so far, not associated with any disease (FD05 and FD08, Figure 1). In another proband, there was a possible disease-causing biallelic genotype (FD07, Figure 2), while, in the last proband, a de novo mosaic variant without strong evidence of pathogenicity was found (FD01, Figure 2). All variants were absent from the ClinVar database and classified with default Varsome settings as variants of uncertain significance (VUSs) by The American College of Medical Genetics and Genomics (ACMG) criteria [35]. Three of them upgraded to "likely pathogenic" when the de novo criterion was included (PS2). These findings are described in detail below (also see Table 2 and Table S2). Technical parameters of WES in trios are shown in Table S1. Table S3, included in Supplementary Materials, also shows all ultra-rare $(<0.00001)$ variants found in probands that could contribute to the disease but were identified as inherited from parent.

\subsubsection{FD05}

In the proband, we found a de novo missense mutation p.Gly257Ser/c.769G $>\mathrm{A}$ in the TRIB3 gene (tribbles pseudokinase 3, MIM \#607898) (Figure 1A). TRIB3 has not been linked to any cardiac disease; however, the amino acid is highly conserved among distant species (Figure 1B). The variant has been found in two subjects from the gnomAD exome database (0.000008). It has seven pathogenic predictions vs. two benign predictions.

The proband was a female with severe exercise intolerance (NYHA class III) at the age of $40 \mathrm{y}$, which followed a flu-like syndrome two months earlier. She had a dilated left ventricle with moderate mitral insufficiency, global diffuse hypokinesis of LVEF 10-15\%, and the coronary CTA was normal. She received a single-chamber implantable cardioverter-defibrillator (ICD-VR) and responded slowly to treatment with LVEF of $35 \%$. At the age of 44 , she is in NYHA II class heart failure.

\subsubsection{FD08}

In the proband, we found a de novo missense mutation p.Arg283His/c.848G $>\mathrm{A}$ in the SLC2A6 gene (solute carrier family 2 member 6, MIM \#606813) (Figure 1A). SLC2A6 has not been associated with any disease. The variant has ambiguous deleteriousness scores (three pathogenic predictions vs. four benign predictions), it was not found in gnomAD, and the amino acid is highly conserved (Figure 1B).

The 29-year-old female proband started having palpitations at $21 \mathrm{y}$, and at $28 \mathrm{y}$, she was diagnosed with eosinophilic myocarditis following a parasitic infestation, on the basis of blood eosinophilia and CMR examination. At $29 \mathrm{y}$, she was found to have complex ventricular arrhythmia and systolic dysfunction with LVEF 38\%, and a subsequent CMR study confirmed diffuse areas of LGE. During the study, her mother's cousin was diagnosed with cardiomyopathy but she was unavailable for the study. 
A
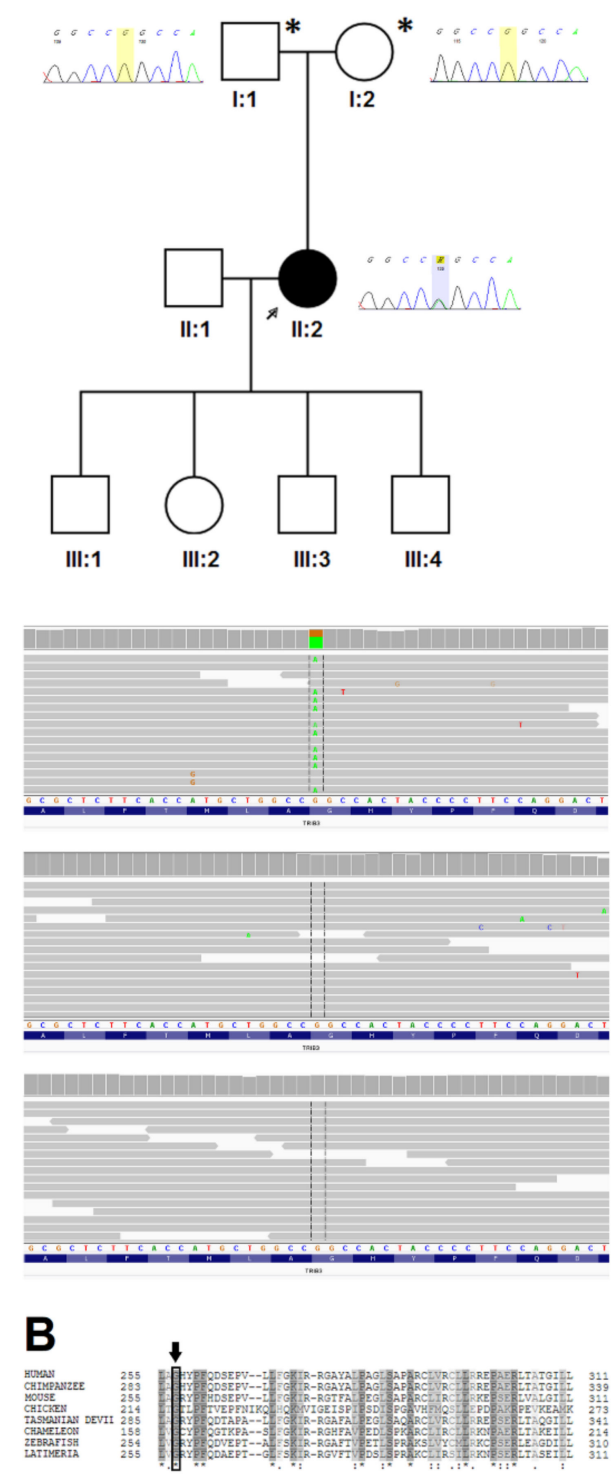

SLC2A6 (FD08)
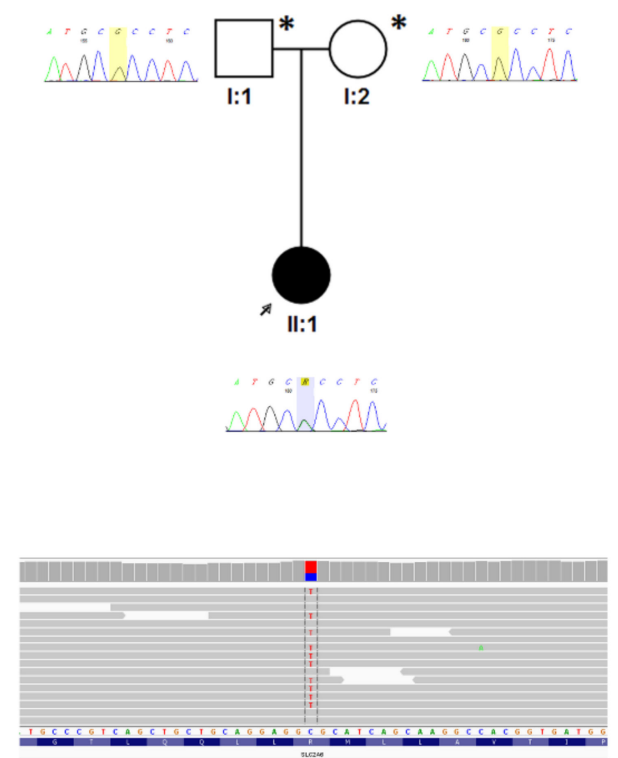

father

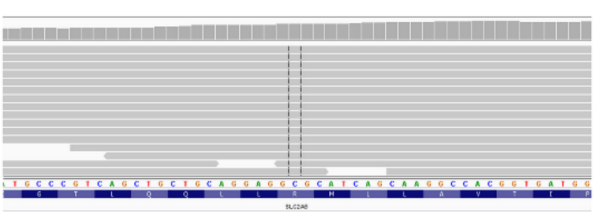

mother
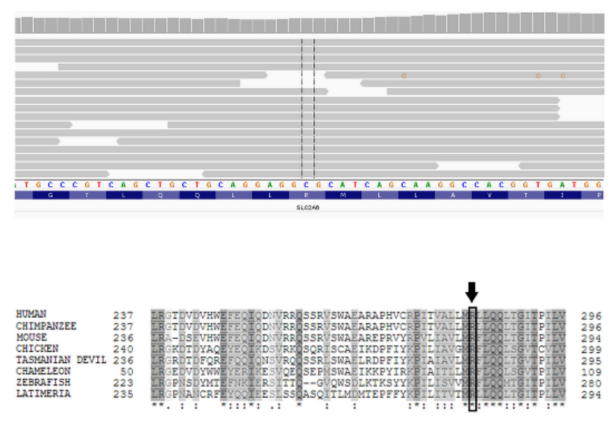

Figure 1. (A) The pedigrees of families with de novo variants in novel cardiomyopathy candidate-genes, corresponding Sanger chromatograms, and IGV views in trios. Left: TRIB3 variant p.Gly257Ser/c.769G >A (family FD05); right: SLC2A6 variant p.Arg283His/c.848G>A (family FD08); (B) Evolutionary conservation of amino acid residues altered by de novo missense mutations in probands. Aligned UniProt sequences for SLC2A6: \#GTR6 (Homo sapiens), \#H2R2P8 (Pan troglodytes), \#A2AR26 (Mus musculus), \#F1NAR8 (Gallus gallus), \#G3VXJ7 (Sarcophilus harrisii), \#H9GLX0 (Anolis carolinensis), \#F1RB41 (Danio rerio), \#H3AT68 (Latimeria chalumnae), and for TRIB3: \#TRIB3 (Homo sapiens), \#H2QJS5 (Pan troglodytes), \#TRIB3 (Mus musculus), \#R4GLD1 (Gallus gallus), \#G3VRY0 (Sarcophilus harrisii), \#H9GDK2 (Anolis carolinensis), \#F1QCV8 (Danio rerio), \#H3AA74 (Latimeria chalumnae).

\subsubsection{FD01}

We found a potential mosaic variant (11\% of reads) p.Arg633Trp/c.1897C $>\mathrm{T}$ in the UNC45A gene (Unc-45 myosin chaperone A, MIM \#611219). The variant was found in 11\% of 277 reads in peripheral blood and it was confirmed by Sanger sequencing (Figure 2). As the proband is deceased, study of other tissues was not performed. The variant has ambiguous predictions of deleteriousness (four pathogenic predictions vs. four benign predictions) and is relatively frequent in the South Asian population (gnomAD $\mathrm{f}=0.00039)$, and also in the homozygous state $(n=1)$. 
The male 36-year-old proband started having palpitations and NYHA class III exercise intolerance at $31 \mathrm{y}$. Three months earlier, he had a flu-like syndrome. He was in sinus rhythm, and diffuse left ventricular dilation and profound hypokinesis were found on the echocardiogram. Coronary angiography was normal. On the 24 hour Holter ECG, frequent ventricular ectopy was present with $>20000$ VEBs. The patient had repeated RF ablation of ventricular arrhythmia, with partial response. At $34 \mathrm{y}$, paroxysmal atrial fibrillation episodes occurred that led to exacerbation of heart failure symptoms. Sinus rhythm had been restored with cardioversion. He died at $36 \mathrm{y}$, probably due to an embolic episode.

\subsubsection{FD07}

Based on copy number variant analysis confirmed by the single-nucleotide variants (SNVs) configuration from the trio analysis, we found evidence in the proband of a CNV removing the $A P O B E C 3 B$ gene (apolipoprotein B mRNA editing enzyme catalytic subunit 3B, MIM \#607110). The deletion was inherited from the mother, unmasking a hemizygous p.Cys217Tyr/c.650G $>A$ variant of paternal origin (Figure 2). The variant has high deleteriousness scores (six pathogenic predictions vs. one benign prediction) and was not found in gnomAD. The patient was also found to have haplotype IV and splice-site SV-154 in the APOBEC3H locus [36]. The APOBEC3H SV-154 variant predicts loss of function due to a loss of multiple conserved structural elements [36].

The female proband had a history of DCM, diagnosed at the age of two. The onset of the disease was associated with a bronchopulmonary infection when the dysfunction of the left ventricle was diagnosed. At $21 \mathrm{y}$, she had ablation of ventricular arrhythmia. At $24 \mathrm{y}$, she was in NYHA class II with significant left ventricular dysfunction (LVEF 30\%, and LVEDD $67 \mathrm{~mm}$ ) and frequent ventricular ectopy with few complex forms on the Holter 24 hour ECG. She refused ICD implantation. At the age of 26 , she is clinically stable.

A
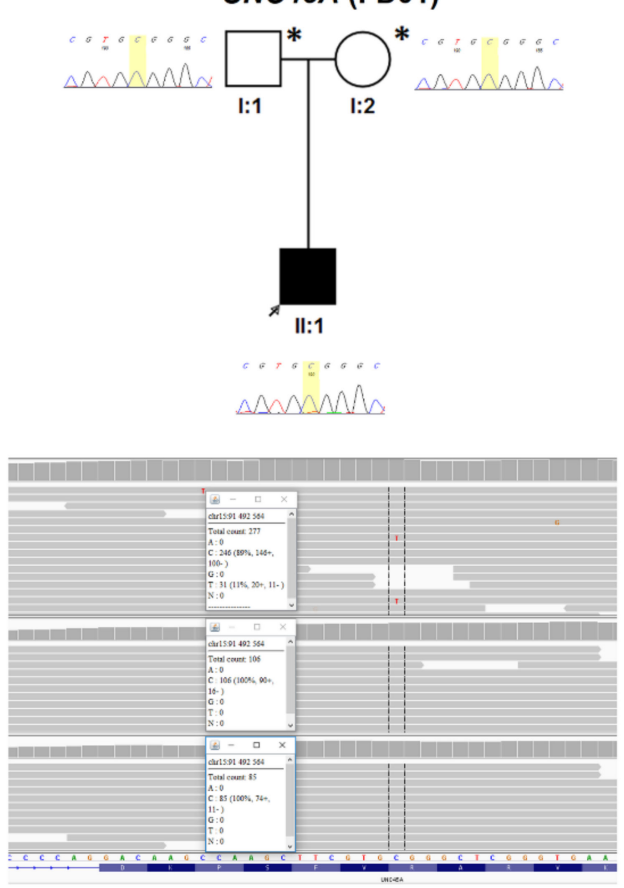

B
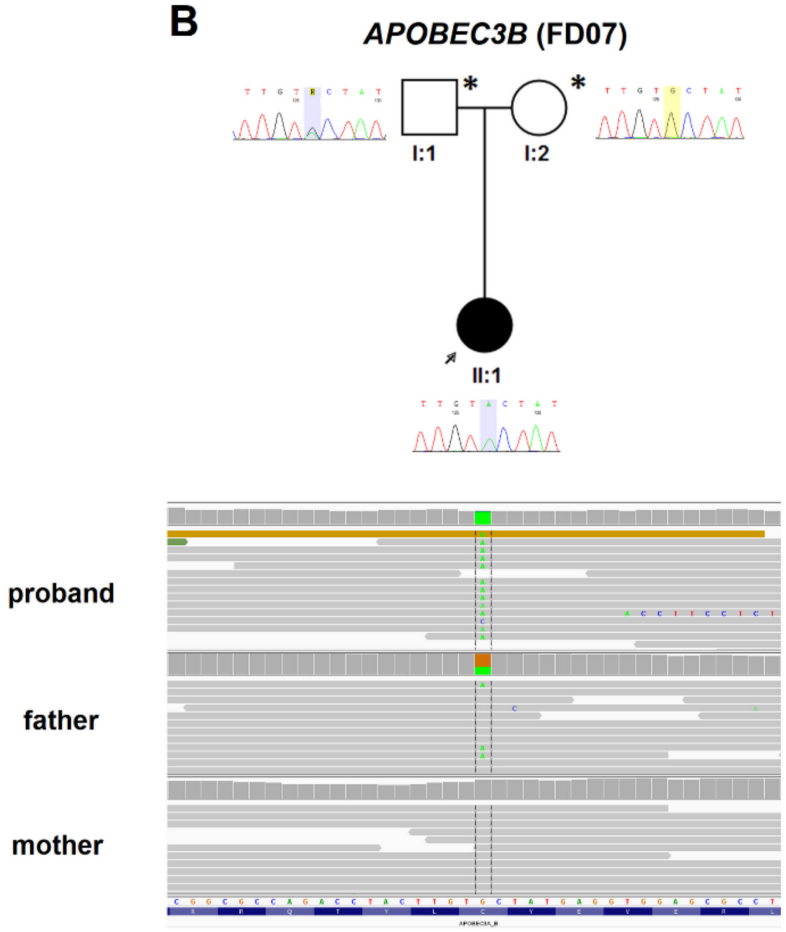

Figure 2. Pedigrees of families with other findings, corresponding Sanger chromatograms, and IGV views in trios. (A) UNC45A possible mosaic de novo variant p.Arg633Trp/c.1897C $>\mathrm{T}$ (11\% of reads and corresponding lower T peak in proband's chromatogram) (Family FD01); (B) APOBEC3B variant p.Cys217Tyr/c.650G>A (hemizygotic variant as a result of maternal copy deletion) (family FD07). 
Table 2. Molecular characteristics of variants of interest in novel genes from whole exome sequencing (WES) analysis in trios found in probands.

\begin{tabular}{|c|c|c|c|c|c|c|}
\hline \multicolumn{3}{|c|}{ Family } & FD05 & FD08 & FD07 & FD01 \\
\hline \multicolumn{3}{|c|}{ Gene/ Transcript } & TRIB3/ NM_021158.4 & SLC2A6/ NM_017585.3 & $\begin{array}{c}\text { APOBEC3B/ } \\
\text { NM_004900.4 }\end{array}$ & UNC45A/ NM_018671.5 \\
\hline \multicolumn{3}{|c|}{ Variant } & p.Gly257Ser/c.769G $>\mathrm{A}^{1}$ & p.Arg283His/c.848G>A ${ }^{1}$ & p.Cys217Tyr/c.650G > A ${ }^{2}$ & p.Arg633Trp/c.1897C > T ${ }^{3}$ \\
\hline \multicolumn{3}{|c|}{ ID } & rs534951995 & ND & ND & rs374670572 \\
\hline \multicolumn{3}{|c|}{ Genomic Coordinates (GRCh38) } & 20:396382-G>A & $9: 133475040-\mathrm{C}>\mathrm{T}$ & 22:38989537-G>A & $15: 90949334-\mathrm{C}>\mathrm{T}$ \\
\hline \multicolumn{3}{|c|}{ ACMG Verdict (default/if de novo) } & VUS/ Likely Pathog & VUS/ Likely Pathog & VUS/ Likely Pathog & VUS/ VUS \\
\hline \multirow{3}{*}{ Allele frequency } & \multicolumn{2}{|c|}{ GnomAD } & $7.98 \mathrm{e}-06$ & 0 & 0 & $3.94 \mathrm{e}-04$ \\
\hline & \multicolumn{2}{|c|}{ 1000G P3 Eur/tot } & $0 / 2.0 \mathrm{e}-04$ & $0 / 0$ & $0 / 0$ & 0/7.99e-04 \\
\hline & \multicolumn{2}{|c|}{ ESP6500 Eur/tot } & $0 / 0$ & $0 / 0$ & $0 / 0$ & $1.20 \mathrm{e}-04 / 8.00 \mathrm{e}-05$ \\
\hline \multirow{8}{*}{ Prediction Scores } & \multirow{5}{*}{ General } & DANN & 0.9988 & 0.9993 & 0.9458 & 0.9979 \\
\hline & & Mutation Taster & Disease causing & Disease causing & Polymorphism & Disease causing \\
\hline & & FATHMM & Tolerated & Tolerated & Damaging & Tolerated \\
\hline & & Meta SVM & Damaging & Tolerated & Damaging & Tolerated \\
\hline & & MetalR & Tolerated & Tolerated & Damaging & Tolerated \\
\hline & \multirow{2}{*}{ Conservation } & GERP & NR 5.269, RS 5.269 & NR 5.3, RS 5.3 & NR 1.919, RS 1.919 & NR 5.28, RS 4.36 \\
\hline & & Mutation Assessor & High & Low & High & Low \\
\hline & Functional & Provean & Damaging & Neutral & Damaging & Damaging \\
\hline
\end{tabular}

\footnotetext{
${ }^{1}$ Variants verified as de novo. ${ }^{2}$ Variant in hemizygous state. ${ }^{3}$ Variant in possible mosaicism. EUR - European; TOT - total, ND - no data, VUS - variant of uncertain significance, Pathog -
} pathogenic, ND - no data. 


\subsection{Parental Age}

In our group, the age of fathers and mothers at the time of probands' birth was relatively low (28.2 \pm 6.3 , range $21-40$; and $24.6 \pm 3.8$, range $18-31$, respectively, Table S4).

\section{Discussion}

The estimated rate for germline de novo single-nucleotide variants in humans is 1.0 to $1.8 \times$ $10^{-8}$ per nucleotide per generation [37]. This means about 44 to 82 variants in the average human genome, with one or two (about 3\%) in the coding sequence [38], although this number varies within and between families [39]. An important aspect of de novo mutations is the reproductive fitness impairment they cause. This was particularly demonstrated for neurodevelopmental diseases, in which de novo mutations are responsible for the relatively high prevalence of these disorders in the general population (reviewed in [40]). Furthermore, in the X-linked Duchenne dystrophy, the percentage of de novo mutations in affected males reaches up to $40 \%$ [41]. According to the ACMG criteria for the pathogenicity of genetic variants, the occurrence of a de novo mutation with both maternity and paternity confirmed (PS2 criterion) is exactly as strong as well-established in vitro or in vivo functional studies (PS3), and both arguments belong to the class of "strong evidence of pathogenicity" [35]. If it is de novo, the variant is usually promoted to a higher class of pathogenicity. Hence, the value of de novo mutation studies is both determining the pathogenicity of variants in known genes, as well as searching for new disease-causing genes.

We present 10 cases of de novo mutations in patients with cardiomyopathy. In 6 of 10 cases of sporadic DCM and in single cases of RCM and HCM, we found de novo mutations in genes previously associated with cardiomyopathy (CRYAB, DSP, MYH7, SCN5A, TNNC1, and TTN). Interestingly, in all but one case, the same variants or variants at the same position have been previously associated with the disease. The exception was $C R Y A B$ p.Thr40Met found in a proband and his daughter who both had cardiac disease and severe myopia. In this family, the variant was inherited by a daughter who - at a relatively young age - was not a healthy person. Given the fact that cardiomyopathy has age- and gender-dependent penetrance and that the female sex often has a milder course of the disease, we assumed this to be evidence of cosegregation, thus suggesting pathogenicity of this variant. Although it is not clear whether this variant causes disease, it is noteworthy that pathogenic $C R Y A B$ variants are associated with cardiomyopathies/myopathies and ocular features such as cataracts (https://omim.org). However, given the limited knowledge on both the CRYAB pathogenic spectrum (only 11 variants are listed in ClinVar) and genetics of myopia, it is intriguing to speculate that the coexistence of dilated cardiomyopathy and myopia observed in the family FD10 may be caused by the $C R Y A B$ variant. In another two cases of sporadic DCM, we found de novo mutations in potential novel DCM candidate genes, TRIB3 and SLC2A6. In the remaining two probands, there was a possible disease-causing biallelic genotype ( $A P O B E C$ genes), and a de novo mosaic variant in the UNC45A gene without strong evidence of pathogenicity was found.

Even though a small study group does not provide reliable quantitative results, the high prevalence of de novo mutations ( $83 \%$ among all cardiomyopathies and $80 \%$ among DCMs) emphasizes that genetic screening is also indicated in cardiomyopathy cases without family history.

Unsurprisingly, one of the de novo variants was found in the TTN gene, which is the main locus for DCM. However, the variant we found was a missense, while TTN association with the disease applies mainly to truncating mutations. Missense TTN variants are abundant, due to the large size of the gene, and are generally considered benign or of uncertain significance (according to ClinVar database only $<1 \%$ of TTN missense variants have pathogenic or likely pathogenic status). However, a de novo TTN missense mutation, especially when transmitted to the affected child, is likely to be pathogenic.

Another important finding is the novel TNNC1 variant. Among troponins, TNNC1 is the only gene whose role in cardiomyopathies is controversial, including a suggestion of the recessive model of inheritance, despite functional studies indicating the pathogenicity of monoallelic variants [42]. In our 
proband, the TNNC1 variant was in the heterozygous state and arose de novo, suggesting a dominant pathogenic effect.

An important aspect of our study is the identification of novel cardiomyopathy candidate genes. It is intriguing that among probands without defects in known genes, we found plausible candidate variants (ultra-low population frequency and in silico pathogenicity prediction) in genes such as $A P O B E C, T R I B 3$, and SLC2A6, which are likely to affect immunological function and/or reaction to stress. Interestingly, all these variants were found in patients in whom the disease was associated with infection (bronchopulmonary infection, flu-like syndrome, or parasitic infestation, respectively).

The $A P O B E C$ genes have a well-established role in innate immunity against retroviral infection and cancers through restricting the replication of viruses and transposons. $A P O B E C 3 B$ is not indispensable, as full gene deletions exist in the general population [43]. However, a deleted $A P O B E C 3 B$ locus together with inactive $A P O B E C 3 H$ haplotypes (i.e., hapIV, which likely has no anti-viral activity at all [44]) may increase the susceptibility to infection by HTLV-1 with severe implications for disease progression [45]. Although p.Cys217Tyr is not a de novo variant, taking into account the infection-related onset of DCM in early childhood, we suggest that the specific genotype/haplotype in the APOBEC3 family (i.e., a single copy of $A P O B E C 3 B$ damaged by the missense variant together with $A P O B E C 3 H$ hapIV) may have contributed to cardiomyopathy in this patient. Furthermore, this observation suggests a link between genetics and myocarditis, especially interesting considering that the history of myocarditis is a risk factor for dilated cardiomyopathy.

TRIB3 is a stress-related gene that induces apoptosis during ER stress [46]. In the heart, TRIB3 has a low expression but is induced by hypoxia [47] or the transcription factor NF-kappaB, which it negatively regulates. In addition, TRIB3 sensitizes cells to TNF- and TRAIL-induced apoptosis and is involved in insulin signaling [48]. In mice, Trib3 knock-out affects mast cell function and beta cell apoptosis (http://www.informatics.jax.org/). Intriguingly, TRIB3 interacts with members of the APOBEC3 family [49].

SLC2A6 encodes a poorly characterized glucose transporter GLUT6 with a likely function in the immune system [50]. In mice, Glut6 is upregulated by activation of T lymphocytes [51], while in humans, the GLUT6 protein concentration was shown to increase in CD4+ T cells on HIV-1 infection [52]. Recently, GLUT6 was shown to be a lysosomal transporter regulated by inflammatory stimuli and modulating glycolysis in macrophages [53] Glut6 knock-out mice had decreased resistance to LPS-induced shock [53].

The UNC45A gene could also be implicated in stress-related pathomechanism as it is a co-chaperone for HSP90 while another DCM-related gene, BAG3, is a co-chaperone for HSP70 [54]. However, the relatively high population prevalence of the variant argues against its major role as a disease cause.

The presence of a de novo mutation in a known gene is strong evidence of its pathogenicity, while in the case of an unknown gene, it only allows us to hypothesize on its possible association with the disease. Clearly, such hypotheses should be verified by functional studies and by identification of additional patients. At present, the role of the variants we found is difficult to evaluate as, to our knowledge, this is the first report of association of the respective genes with DCM. However, given the involvement of these candidate genes in immune response and an established association between DCM and inflammation, we think that our findings offer a valuable clue for further studies.

\section{Limitations}

The estimate of de novo mutation prevalence in this study is likely overestimated due to the small number and selection of probands as, even in typical familial cardiomyopathies, the prevalence of identified mutations does not exceed $40 \%$ in DCM [55] and approx. 65\% in HCM [56]. The detection of mutations was limited to the coding sequence, and the CNV analysis performed did not allow us to detect other types of structural variants such as inversions, balanced translocations, or complex rearrangements. The lack of functional analyses does not allow us to confirm the hypothetical role of the identified genes and their association with the disease. 


\section{Conclusions}

The high percentage of de novo mutations observed in our cohort suggests that their occurrence in sporadic cases of cardiomyopathy is non-negligible. De novo variants detected in patients without an established cause for disease raises the possibility that genes involved in immune/stress response such as APOBEC, TRIB3, or SLC2A6 could be relevant for cardiomyopathy development, especially in patients with disease onset associated with infection/infestation. However, these hypotheses need to be verified by further studies, functional analyses, and by identification of additional patients with mutations in respective genes, as the role of the variants we found is difficult to evaluate based only on their de novo origin.

Supplementary Materials: The following are available online at http://www.mdpi.com/2077-0383/9/2/370/s1, Figure S1: The pedigrees of families with de novo variants in known cardiomyopathy genes and corresponding Sanger chromatograms, Table S1: Technical parameters of WES in trios, Table S2: Detailed clinical characteristics of probands, Table S3: Other rare $(<0.00001$ in gnomAD) variants identified in probands as inherited from a parent, Table S4: The ages of parents of probands' with de novo mutations.

Author Contributions: Conceptualization, M.F.; Funding acquisition, M.F. and R.P.; Investigation, M.F., G.T., M.R., J.K., A.B., G.K. and P.K.; Project administration, M.F. and R.P.; Resources, P.C., T.R., M.S., M.S.-W., M.B., T.Z. and Z.T.B.; Software, P.S.; Supervision, M.F. and R.P.; Validation, G.T., M.R., J.K., A.B., G.K., P.K. and A.L.; Visualization, M.F. and P.S.; Writing-Original draft, M.F. and Z.T.B.; Writing - Review \& editing, M.F., A.L., Z.T.B. and R.P. All authors have read and agreed to the published version of the manuscript.

Funding: This research was funded by Narodowe Centrum Nauki (Poland), grant number 2013/11/N/NZ2/02528. Zofia Bilinska's work is supported by the ERA-CVD grant: DETECTIN-HF.

Acknowledgments: The authors would like to thank the patients for their help and cooperation, which have greatly contributed to this article.

Conflicts of Interest: The authors declare no conflict of interest. The funders had no role in the design of the study; in the collection, analyses, or interpretation of data; in the writing of the manuscript or in the decision to publish the results.

\section{References}

1. Elliott, P.; Andersson, B.; Arbustini, E.; Bilinska, Z.; Cecchi, F.; Charron, P.; Dubourg, O.; Kühl, U.; Maisch, B.; McKenna, W.J.; et al. Classification of the cardiomyopathies: A position statement from the European Society of Cardiology Working Group on Myocardial and Pericardial Diseases. Eur. Heart J. 2008, 29, 270-276. [CrossRef]

2. Kimura, A. Molecular genetics and pathogenesis of cardiomyopathy. J. Hum. Genet. 2016, 61, 41-50. [CrossRef]

3. Keller, D.I.; Stepowski, D.; Balmer, C.; Simon, F.; Guenthard, J.; Bauer, F.; Itin, P.; David, N.; Drouin-Garraud, V.; Fressart, V. De novo heterozygous desmoplakin mutations leading to Naxos-Carvajal disease. Swiss Med. Wkly. 2012, 142, w13670. [CrossRef] [PubMed]

4. Boyden, L.M.; Kam, C.Y.; Hernández-Martín, A.; Zhou, J.; Craiglow, B.G.; Sidbury, R.; Mathes, E.F.; Maguiness, S.M.; Crumrine, D.A.; Williams, M.L.; et al. Dominant de novo DSP mutations cause erythrokeratodermia-cardiomyopathy syndrome. Hum. Mol. Genet. 2016, 25, 348-357. [CrossRef] [PubMed]

5. Sugie, K.; Yoshizawa, H.; Onoue, K.; Nakanishi, Y.; Eura, N.; Ogawa, M.; Nakano, T.; Sakaguchi, Y.; Hayashi, Y.K.; Kishimoto, T.; et al. Early onset of cardiomyopathy and intellectual disability in a girl with Danon disease associated with a de novo novel mutation of the LAMP2 gene. Neuropathol. Off. J. Jpn. Soc. Neuropathol. 2016, 36, 561-565. [CrossRef] [PubMed]

6. Liu, Y.; Bai, R.; Wang, L.; Zhang, C.; Zhao, R.; Wan, D.; Chen, X.; Caceres, G.; Barr, D.; Barajas-Martinez, H.; et al. Identification of a novel de novo mutation associated with PRKAG2 cardiac syndrome and early onset of heart failure. PLoS ONE 2013, 8, e64603. [CrossRef]

7. Xu, Y.; Gray, A.; Hardie, D.G.; Uzun, A.; Shaw, S.; Padbury, J.; Phornphutkul, C.; Tseng, Y.-T. A novel, de novo mutation in the PRKAG2 gene: Infantile-onset phenotype and the signaling pathway involved. Am. J. Physiol. Heart Circ. Physiol. 2017, 313, H283-H292. [CrossRef] 
8. Harms, F.L.; Alawi, M.; Amor, D.J.; Tan, T.Y.; Cuturilo, G.; Lissewski, C.; Brinkmann, J.; Schanze, D.; Kutsche, K.; Zenker, M. The novel RAF1 mutation p.(Gly361Ala) located outside the kinase domain of the CR3 region in two patients with Noonan syndrome, including one with a rare brain tumor. Am. J. Med. Genet. A. 2018, 176, 470-476. [CrossRef]

9. Yoo, T.Y.; Kim, M.R.; Son, J.S.; Lee, R.; Bae, S.H.; Chung, S.; Kim, K.S.; Seong, M.-W.; Park, S.S. Identification of a Novel De Novo Mutation of the TAZ Gene in a Korean Patient with Barth Syndrome. J. Cardiovasc. Ultrasound 2016, 24, 153-157. [CrossRef]

10. Long, P.A.; Zimmermann, M.T.; Kim, M.; Evans, J.M.; Xu, X.; Olson, T.M. De novo RRAGC mutation activates mTORC1 signaling in syndromic fetal dilated cardiomyopathy. Hum. Genet. 2016, 135, 909-917. [CrossRef]

11. Guo, X.; Ling, C.; Liu, Y.; Zhang, X.; Zhang, S. A Case of Novel Lamin A/C Mutation Manifesting as Atypical Progeroid Syndrome and Cardiomyopathy. Can. J. Cardiol. 2016, 32, 1166.e29-1166.e31. [CrossRef] [PubMed]

12. Olson, T.M.; Doan, T.P.; Kishimoto, N.Y.; Whitby, F.G.; Ackerman, M.J.; Fananapazir, L. Inherited and de novo mutations in the cardiac actin gene cause hypertrophic cardiomyopathy. J. Mol. Cell. Cardiol. 2000, 32, 1687-1694. [CrossRef]

13. Watkins, H.; Thierfelder, L.; Hwang, D.S.; McKenna, W.; Seidman, J.G.; Seidman, C.E. Sporadic hypertrophic cardiomyopathy due to de novo myosin mutations. J. Clin. Invest. 1992, 90, 1666-1671. [CrossRef] [PubMed]

14. Karam, S.; Raboisson, M.-J.; Ducreux, C.; Chalabreysse, L.; Millat, G.; Bozio, A.; Bouvagnet, P. A de novo mutation of the beta cardiac myosin heavy chain gene in an infantile restrictive cardiomyopathy. Congenit. Heart Dis. 2008, 3, 138-143. [CrossRef] [PubMed]

15. Okada, S.; Suzuki, Y.; Arimura, T.; Kimura, A.; Narumi, H.; Hasegawa, S. A novel de novo mutation of $\beta$-cardiac myosin heavy chain gene found in a twelve-year-old boy with hypertrophic cardiomyopathy. $J$. Genet. 2014, 93, 557-560. [CrossRef] [PubMed]

16. Zhao, P.; Cui, H.-L.; He, T.-T.; Wang, J.-G.; Wang, D.; Feng, X.-X.; Zou, Y.-B.; Wang, Y.-L.; Wang, J.-Z.; Hui, R.-T.; et al. Familial hypertrophic cardiomyopathy caused by a de novo Gly716Arg mutation of the $\beta$-myosin heavy chain. Cardiol. Young 2017, 27, 467-472. [CrossRef]

17. Mouton, J.M.; Pellizzon, A.S.; Goosen, A.; Kinnear, C.J.; Herbst, P.G.; Brink, P.A.; Moolman-Smook, J.C. Diagnostic disparity and identification of two TNNI3 gene mutations, one novel and one arising de novo, in South African patients with restrictive cardiomyopathy and focal ventricular hypertrophy. Cardiovasc. J. Afr. 2015, 26, 63-69. [CrossRef]

18. Peddy, S.B.; Vricella, L.A.; Crosson, J.E.; Oswald, G.L.; Cohn, R.D.; Cameron, D.E.; Valle, D.; Loeys, B.L. Infantile restrictive cardiomyopathy resulting from a mutation in the cardiac troponin $\mathrm{T}$ gene. Pediatrics 2006, 117, 1830-1833. [CrossRef]

19. Long, P.A.; Larsen, B.T.; Evans, J.M.; Olson, T.M. Exome Sequencing Identifies Pathogenic and Modifier Mutations in a Child With Sporadic Dilated Cardiomyopathy. J. Am. Heart Assoc. 2015, 4. [CrossRef]

20. Watkins, H.; Anan, R.; Coviello, D.A.; Spirito, P.; Seidman, J.G.; Seidman, C.E. A de novo mutation in alpha-tropomyosin that causes hypertrophic cardiomyopathy. Circulation 1995, 91, 2302-2305. [CrossRef]

21. Kelle, A.M.; Bentley, S.J.; Rohena, L.O.; Cabalka, A.K.; Olson, T.M. Ebstein anomaly, left ventricular non-compaction, and early onset heart failure associated with a de novo $\alpha$-tropomyosin gene mutation. Am. J. Med. Genet. A 2016, 170, 2186-2190. [CrossRef] [PubMed]

22. Petersen, S.E.; Selvanayagam, J.B.; Wiesmann, F.; Robson, M.D.; Francis, J.M.; Anderson, R.H.; Watkins, H.; Neubauer, S. Left ventricular non-compaction: insights from cardiovascular magnetic resonance imaging. J. Am. Coll. Cardiol. 2005, 46, 101-105. [CrossRef] [PubMed]

23. Ploski, R.; Pollak, A.; Müller, S.; Franaszczyk, M.; Michalak, E.; Kosinska, J.; Stawinski, P.; Spiewak, M.; Seggewiss, H.; Bilinska, Z.T. Does p.Q247X in TRIM63 cause human hypertrophic cardiomyopathy? Circ. Res. 2014, 114, e2-e5. [CrossRef] [PubMed]

24. Talevich, E.; Shain, A.H.; Botton, T.; Bastian, B.C. CNVkit: Genome-Wide Copy Number Detection and Visualization from Targeted DNA Sequencing. PLoS Comput. Biol. 2016, 12, e1004873. [CrossRef] [PubMed]

25. Gerull, B.; Gramlich, M.; Atherton, J.; McNabb, M.; Trombitás, K.; Sasse-Klaassen, S.; Seidman, J.G.; Seidman, C.; Granzier, H.; Labeit, S.; et al. Mutations of TTN, encoding the giant muscle filament titin, cause familial dilated cardiomyopathy. Nat. Genet. 2002, 30, 201-204. [CrossRef]

26. Hinson, J.T.; Chopra, A.; Nafissi, N.; Polacheck, W.J.; Benson, C.C.; Swist, S.; Gorham, J.; Yang, L.; Schafer, S.; Sheng, C.C.; et al. HEART DISEASE. Titin mutations in iPS cells define sarcomere insufficiency as a cause of dilated cardiomyopathy. Science 2015, 349, 982-986. [CrossRef] 
27. Castelletti, S.; Vischer, A.S.; Syrris, P.; Crotti, L.; Spazzolini, C.; Ghidoni, A.; Parati, G.; Jenkins, S.; Kotta, M.-C.; McKenna, W.J.; et al. Desmoplakin missense and non-missense mutations in arrhythmogenic right ventricular cardiomyopathy: Genotype-phenotype correlation. Int. J. Cardiol. 2017, 249, 268-273. [CrossRef]

28. Kapplinger, J.D.; Tester, D.J.; Alders, M.; Benito, B.; Berthet, M.; Brugada, J.; Brugada, P.; Fressart, V.; Guerchicoff, A.; Harris-Kerr, C.; et al. An international compendium of mutations in the SCN5A-encoded cardiac sodium channel in patients referred for Brugada syndrome genetic testing. Heart Rhythm 2010, 7, 33-46. [CrossRef]

29. Wang, C.; Hata, Y.; Hirono, K.; Takasaki, A.; Ozawa, S.W.; Nakaoka, H.; Saito, K.; Miyao, N.; Okabe, M.; Ibuki, K.; et al. A Wide and Specific Spectrum of Genetic Variants and Genotype-Phenotype Correlations Revealed by Next-Generation Sequencing in Patients with Left Ventricular Noncompaction. J. Am. Heart Assoc. 2017, 6. [CrossRef]

30. Villard, E.; Duboscq-Bidot, L.; Charron, P.; Benaiche, A.; Conraads, V.; Sylvius, N.; Komajda, M. Mutation screening in dilated cardiomyopathy: prominent role of the beta myosin heavy chain gene. Eur. Heart J. 2005, 26, 794-803. [CrossRef]

31. García-Giustiniani, D.; Arad, M.; Ortíz-Genga, M.; Barriales-Villa, R.; Fernández, X.; Rodríguez-García, I.; Mazzanti, A.; Veira, E.; Maneiro, E.; Rebolo, P.; et al. Phenotype and prognostic correlations of the converter region mutations affecting the $\beta$ myosin heavy chain. Heart Br. Card. Soc. 2015, 101, 1047-1053. [CrossRef]

32. Perrot, A.; Schmidt-Traub, H.; Hoffmann, B.; Prager, M.; Bit-Avragim, N.; Rudenko, R.I.; Usupbaeva, D.A.; Kabaeva, Z.; Imanov, B.; Mirrakhimov, M.M.; et al. Prevalence of cardiac beta-myosin heavy chain gene mutations in patients with hypertrophic cardiomyopathy. J. Mol. Med. Berl. Ger. 2005, 83, 468-477. [CrossRef] [PubMed]

33. Kubo, T.; Gimeno, J.R.; Bahl, A.; Steffensen, U.; Steffensen, M.; Osman, E.; Thaman, R.; Mogensen, J.; Elliott, P.M.; Doi, Y.; et al. Prevalence, clinical significance, and genetic basis of hypertrophic cardiomyopathy with restrictive phenotype. J. Am. Coll. Cardiol. 2007, 49, 2419-2426. [CrossRef] [PubMed]

34. Van Spaendonck-Zwarts, K.Y.; van Rijsingen, I.A.W.; van den Berg, M.P.; Lekanne Deprez, R.H.; Post, J.G.; van Mil, A.M.; Asselbergs, F.W.; Christiaans, I.; van Langen, I.M.; Wilde, A.A.M.; et al. Genetic analysis in 418 index patients with idiopathic dilated cardiomyopathy: overview of 10 years' experience. Eur. J. Heart Fail. 2013, 15, 628-636. [CrossRef] [PubMed]

35. Richards, S.; Aziz, N.; Bale, S.; Bick, D.; Das, S.; Gastier-Foster, J.; Grody, W.W.; Hegde, M.; Lyon, E.; Spector, E.; et al. Standards and guidelines for the interpretation of sequence variants: a joint consensus recommendation of the American College of Medical Genetics and Genomics and the Association for Molecular Pathology. Genet. Med. Off. J. Am. Coll. Med. Genet. 2015, 17, 405-424. [CrossRef] [PubMed]

36. Shaban, N.M.; Shi, K.; Lauer, K.V.; Carpenter, M.A.; Richards, C.M.; Salamango, D.; Wang, J.; Lopresti, M.W.; Banerjee, S.; Levin-Klein, R.; et al. The Antiviral and Cancer Genomic DNA Deaminase APOBEC3H Is Regulated by an RNA-Mediated Dimerization Mechanism. Mol. Cell 2018, 69, 75-86.e9. [CrossRef]

37. Kong, A.; Frigge, M.L.; Masson, G.; Besenbacher, S.; Sulem, P.; Magnusson, G.; Gudjonsson, S.A.; Sigurdsson, A.; Jonasdottir, A.; Jonasdottir, A.; et al. Rate of de novo mutations and the importance of father's age to disease risk. Nature 2012, 488, 471-475. [CrossRef]

38. Francioli, L.C.; Polak, P.P.; Koren, A.; Menelaou, A.; Chun, S.; Renkens, I.; van Duijn, C.M.; Swertz, M.; Wijmenga, C.; Genome of the Netherlands Consortium; et al. Genome-wide patterns and properties of de novo mutations in humans. Nat. Genet. 2015, 47, 822-826. [CrossRef]

39. Conrad, D.F.; Keebler, J.E.M.; DePristo, M.A.; Lindsay, S.J.; Zhang, Y.; Casals, F.; Idaghdour, Y.; Hartl, C.L.; Torroja, C.; Garimella, K.V.; et al. Variation in genome-wide mutation rates within and between human families. Nat. Genet. 2011, 43, 712-714.

40. Gauthier, J.; Rouleau, G.A. De novo mutations in neurological and psychiatric disorders: effects, diagnosis and prevention. Genome Med. 2012, 4, 71. [CrossRef]

41. Chen, W.-J.; Lin, Q.-F.; Zhang, Q.-J.; He, J.; Liu, X.-Y.; Lin, M.-T.; Murong, S.-X.; Liou, C.-W.; Wang, N. Molecular analysis of the dystrophin gene in 407 Chinese patients with Duchenne/Becker muscular dystrophy by the combination of multiplex ligation-dependent probe amplification and Sanger sequencing. Clin. Chim. Acta Int. J. Clin. Chem. 2013, 423, 35-38. [CrossRef] [PubMed] 
42. Ploski, R.; Rydzanicz, M.; Ksiazczyk, T.M.; Franaszczyk, M.; Pollak, A.; Kosinska, J.; Michalak, E.; Stawinski, P.; Ziolkowska, L.; Bilinska, Z.T.; et al. Evidence for troponin C (TNNC1) as a gene for autosomal recessive restrictive cardiomyopathy with fatal outcome in infancy. Am. J. Med. Genet. A 2016, 170, 3241-3248. [CrossRef] [PubMed]

43. Kidd, J.M.; Newman, T.L.; Tuzun, E.; Kaul, R.; Eichler, E.E. Population stratification of a common APOBEC gene deletion polymorphism. PLoS Genet. 2007, 3, e63. [CrossRef] [PubMed]

44. Zhen, A.; Du, J.; Zhou, X.; Xiong, Y.; Yu, X.-F. Reduced APOBEC3H variant anti-viral activities are associated with altered RNA binding activities. PLoS ONE 2012, 7, e38771. [CrossRef]

45. Ooms, M.; Krikoni, A.; Kress, A.K.; Simon, V.; Münk, C. APOBEC3A, APOBEC3B, and APOBEC3H haplotype 2 restrict human T-lymphotropic virus type 1. J. Virol. 2012, 86, 6097-6108. [CrossRef]

46. Ohoka, N.; Yoshii, S.; Hattori, T.; Onozaki, K.; Hayashi, H. TRB3, a novel ER stress-inducible gene, is induced via ATF4-CHOP pathway and is involved in cell death. EMBO J. 2005, 24, 1243-1255. [CrossRef]

47. Cheng, W.-P.; Lo, H.-M.; Wang, B.-W.; Chua, S.-K.; Lu, M.-J.; Shyu, K.-G. Atorvastatin alleviates cardiomyocyte apoptosis by suppressing TRB3 induced by acute myocardial infarction and hypoxia. J. Formos. Med. Assoc. Taiwan Yi Zhi 2017, 116, 388-397. [CrossRef]

48. Du, K.; Herzig, S.; Kulkarni, R.N.; Montminy, M. TRB3: a tribbles homolog that inhibits Akt/PKB activation by insulin in liver. Science 2003, 300, 1574-1577. [CrossRef]

49. Aynaud, M.-M.; Suspène, R.; Vidalain, P.-O.; Mussil, B.; Guétard, D.; Tangy, F.; Wain-Hobson, S.; Vartanian, J.-P. Human Tribbles 3 protects nuclear DNA from cytidine deamination by APOBEC3A. J. Biol. Chem. 2012, 287, 39182-39192. [CrossRef]

50. Doege, H.; Bocianski, A.; Joost, H.G.; Schürmann, A. Activity and genomic organization of human glucose transporter 9 (GLUT9), a novel member of the family of sugar-transport facilitators predominantly expressed in brain and leucocytes. Biochem. J. 2000, 350 Pt 3, 771-776. [CrossRef]

51. Macintyre, A.N.; Gerriets, V.A.; Nichols, A.G.; Michalek, R.D.; Rudolph, M.C.; Deoliveira, D.; Anderson, S.M.; Abel, E.D.; Chen, B.J.; Hale, L.P.; et al. The glucose transporter Glut1 is selectively essential for CD4 T cell activation and effector function. Cell Metab. 2014, 20, 61-72. [CrossRef] [PubMed]

52. Kavanagh Williamson, M.; Coombes, N.; Juszczak, F.; Athanasopoulos, M.; Khan, M.B.; Eykyn, T.R.; Srenathan, U.; Taams, L.S.; Dias Zeidler, J.; Da Poian, A.T.; et al. Upregulation of Glucose Uptake and Hexokinase Activity of Primary Human CD4+ T Cells in Response to Infection with HIV-1. Viruses 2018, 10, 114. [CrossRef] [PubMed]

53. Maedera, S.; Mizuno, T.; Ishiguro, H.; Ito, T.; Soga, T.; Kusuhara, H. GLUT6 is a lysosomal transporter that is regulated by inflammatory stimuli and modulates glycolysis in macrophages. FEBS Lett. 2019, 593, 195-208. [CrossRef] [PubMed]

54. Takayama, S.; Xie, Z.; Reed, J.C. An evolutionarily conserved family of Hsp70/Hsc70 molecular chaperone regulators. J. Biol. Chem. 1999, 274, 781-786. [CrossRef] [PubMed]

55. Hershberger, R.E.; Hedges, D.J.; Morales, A. Dilated cardiomyopathy: the complexity of a diverse genetic architecture. Nat. Rev. Cardiol. 2013, 10, 531-547. [CrossRef]

56. Biagini, E.; Olivotto, I.; Iascone, M.; Parodi, M.I.; Girolami, F.; Frisso, G.; Autore, C.; Limongelli, G.; Cecconi, M.; Maron, B.J.; et al. Significance of sarcomere gene mutations analysis in the end-stage phase of hypertrophic cardiomyopathy. Am. J. Cardiol. 2014, 114, 769-776. [CrossRef]

(C) 2020 by the authors. Licensee MDPI, Basel, Switzerland. This article is an open access article distributed under the terms and conditions of the Creative Commons Attribution (CC BY) license (http://creativecommons.org/licenses/by/4.0/). 